\title{
Effet du sésame (Sesamum indicum L.) sur le développement de Striga hermonthica (Del.) Benth.
}

\author{
Amadou Mounkaila Hamissou, Amoukou Adamou Ibrahim, Zangui Hamissou \\ Département de production végétale, Faculté d'Agronomie / Université Abdou Moumouni de Niamey, BP. 10960, E- \\ mail : amadoumounkailah@gmail.com, ibramouk@yahoo.fr, Zanguiagro@gmail.com
}

Original submitted in on $9^{\text {th }}$ June 2020. Published online at www.m.elewa.org/journals/ on $31^{\text {st }}$ August 2020 https://doi.org/10.35759/JABs.152.10

\begin{abstract}
RESUME
Objectif : L'objectif est d'étudier l'effet de sésame sur le développement de Striga hermonthica sur le mil. Méthodologie et résultats : Deux tests de germination in vitro et en pots sont utilisés à cet effet. Les résultats du test in vitro montrent que Striga hermonthica germe bien sur les deux espèces (mil et sésame). L'association du sésame avec le mil, réduit significativement de moitié (avec P-value de 0,01 ) le taux de germination des graines de Striga hermonthica par rapport au témoin (culture pure du mil). Le test de germination en pots montre que les variétés de sésame (HC110 et ICN130) en association avec le mil, diminuent considérablement le nombre de plants de Striga hermonthica émergés, contrairement à d'autres variétés tels que Birkan, ICN137, HC108, EF147, EF146 et HB168. Les variétés de sésame Vgr 156 et 381-7semblent même favoriser son émergence.

Conclusion et application des résultats : Le sésame se comporte comme un faux hôte (une plante qui provoque la germination suicide des graines de striga) pour Striga hermonthica, mais son efficacité dépend de la variété de sésame utilisée, d'où l'intérêt de cibler les plus efficaces. Les meilleures variétés de sésame identifiées comme faux hôtes sont $\mathrm{HC} 110$ et ICN130. Ces variétés de sésame peuvent être utilisées en association avec le mil pour diminuer efficacement le stock de graines de striga dans les champs.

Mots clés : Sesamum indicum, Striga hermonthica, plante piège, Pennisetum glaucum, Niamey.
\end{abstract}

Effect of sesame (Sesamum indicum L.) on the development of Striga hermonthica (Del.) Benth.

\begin{abstract}
Objective: The goal is to study the sesame effect on the development of Striga hermonthica on millet.

Methodology and results: Two germination tests in vitro and in pots are used for this purpose. The in vitro test findings show that the Striga hermonthica germinates well on the two species (millet and sesame). The association of sesame with millet significantly reduces by half (with P-value of 0.01 ) the germination rate of Striga hermonthica seeds compared to the control (pure cultivation of millet). The germination test in pots shows that the varieties of sesame ( $\mathrm{HC110}$ and ICN130) in association with millet considerably decrease the number of emerged plants of Striga hermonthica, unlike other varieties such as Birkan, ICN137, HC108, EF147, EF146 and HB168. The sesame varieties Vgr 156 and 38-1-7 even seems to encourage its emergence.

Conclusion and application of results: Sesame behaves like a false host (a plant that causes suicide germination of striga seeds) for Striga hermonthica, but its effectiveness depends on the variety of sesame
\end{abstract}


used, hence the interest in targeting the most effective. The best sesame varieties identified as false hosts are $\mathrm{HC} 110$ and ICN130. These varieties of sesame can be used in combination whith millet to effectively decrease striga seed strorage in the fields.

Key words: Sesamum indicum, Striga hermonthica, trap plant, Pennisetum glaucum, Niamey.

\section{INTRODUCTION}

Les plantes parasites phanérogames tels que Striga hermonthica se nourrissent de la sève de leurs hôtes (Ouédraogo et al., 2018). Elles prélèvent les éléments nutritifs nécessaires à leurs besoins et ainsi créent un déséquilibre nutritif chez leurs hôtes (Olivier, 1995a, n.d; Bamba et al., 2019). Striga hermonthica, étant une plante parasite des céréales, cause des dégâts énormes sur le mil. En Afrique, en général, les pertes de rendement causées par Striga hermonthica s'estiment à 7 milliards de Dollars américain (Mboob, 1986). En Afrique de l'Ouest, en particulier, les pertes occasionnées varient de 30 à $75 \%$ au niveau national et peuvent aller jusqu'à $100 \%$ au niveau d'une parcelle soit l'équivalent de 2 milliards de Dollar américain par an (Kgosi et al., 2012a ; Spallek et al., 2013). A cet effet, plusieurs méthodes de lutte (mécanique, physique, chimique et culturale) sont testées sans succès (Olivier, 1995b, 1996 ; Showemimo et al., 2002 ; Boussim et al., 2012), avec des insuffisances d'ordre économique,

\section{MATERIELS ET METHODES}

Le matériel végétal est constitué de dix (10) variétés de sésame (HC110; HC108; ICN130; ICN137; EF146 ; EF147 ; Birkan ; 38-1-7 ; Vgr 156 et HB168) (Boureima et al., 2012), d'une variété de mil HKP sensible au Striga et du Striga hermonthica récolté dans les champs de la région de Niamey en 2016.

Méthodologie : Deux tests de germinations sont utilisés dans cette étude.

Test de germination in vitro de Striga hermonthica en présence du mil et du sésame ; L'essai est conduit dans le laboratoire de biotechnologie de l'Institut de Radio Isotope de l'Université Abdou Moumouni. Les graines de mil, de sésame et de Striga hermonthica sont traitées dans une solution d'eau de javel (concentration d'eau de javel brute : $4^{\circ}$ ) à la concentration de $5 \mathrm{ml}$ pour $100 \mathrm{ml}$ d'eau distillée pendant $3 \mathrm{mn}$ pour les stériliser, puis rincées avec de l'eau distillée et stérilisée. La germination est faite dans des boites de pétris stérilisées. Dans chaque boite 10 graines de mil, 10 sociale et environnementale (Olupot et al., 2003 ; Zia and Khan, 2004). La lutte biologique s'aborde de nos jours sous plusieurs aspects, de l'utilisation des microorganismes (bactéries, champignons, virus) jusqu'à l'utilisation des organismes (plante piège ou faux hôte) (Stoop et al., 1981 ; Waters et al., 2017 ; Kokubun, 2018). La lutte biologique contre Striga hermonthica occupe une place importante en malherbologie dans la gestion intégrée des plantes parasites (Rath et al., 2006 ; Lawane et al., 2009). Au Niger, les paysans possèdent des savoirs endogènes dans la lutte contre cette plante parasite redoutable. Et parmi ces savoirs, l'utilisation de sésame (Sesamum undicum L.) comme plante piège est fréquente en milieu rural. Cependant, on connait mal les mécanismes mises en jeux par cette plante. C'est pourquoi cette étude vise à élucider l'action du sésame sur le développement du Striga hermonthica (Del.) Benth. " sur le mil, afin de proposer une méthode de lutte biologique efficace.

graines de sésame et $0,3 \mathrm{~g}$ de Striga hermonthica sont mis en germination. Les graines sont réparties sur $d u$ papier buvard, imbibé de $2 \mathrm{ml}$ d'eau distillée et stérilisée. $2 \mathrm{ml}$ d'eau ont été apportés tous les jours par boite. Ainsi, trois traitements (sésame + Striga hermonthica ; mil + Striga hermonthica, et mil + sésame + Striga hermonthica) sont testés dans un dispositif expérimental en randomisation totale à quatre (4) répétitions. Les boites sont placées dans un incubateur à obscurité, réglé à une température de $25^{\circ} \mathrm{C}$, pendant trois (3) jours. Après 3 jours, l'incubateur est réglé à $11 \mathrm{~h}$ de lumière et $13 \mathrm{~h}$ d'obscurité avec une température de $25^{\circ} \mathrm{C}$.

Observations : Les observations quotidiennes ont porté sur le comptage du nombre de graines germées de Striga hermonthica sur les racines du mil ou du sésame à partir du 4 èm jour, sous une hotte à flux laminaire et en conditions aseptiques à l'aide d'un microscope photonique. 
Test de germination en pots de culture : Un essai en pots est conduit sur le terrain expérimental de la Faculté d'Agronomie, dans un dispositif expérimental en randomisation totale et à 3 répétitions. Dans cet essai, l'effet de dix variétés de sésames, en culture pure et en associations avec le mil a été testé pour voir leur influence sur le développement de Striga hermonthica. Chaque pot contient un volume de $13 \mathrm{~L}$ de substrat $(2 / 3$ de sable prélevé à $2 \mathrm{~m}$ de profondeur et $1 / 3$ de fumier). Une quantité de $3 \mathrm{~g}$ de Striga hermonthica est appliquée dans chaque pot avant le semis. Une quantité de $2 \mathrm{~g}$ d'engrais NPK (15-15-15) est appliquée, après le semis, en micro-dose dans chaque pot. $2 \mathrm{~L}$ d'eau / pot est apportée chaque 2 jours jusqu'à la levée. Au stade 4 feuilles de sésame, un démariage à 3 plants de chaque

\section{RESULTATS}

Le test de germination in vitro montre que Striga hermonthica germe aussi bien sur les radicules du mil pur que sur celles du sésame. La figure 1 (1a, $1 \mathrm{~b}$ et $1 \mathrm{c}$ ) espèce est effectué. Et la fréquence d'irrigation est ramené tous les jours avec une quantité d'eau de $1 \mathrm{~L} /$ pot.

Observations : Elles ont porté sur le nombre de plants de Striga hermonthica émergés dans chaque pot (tous les 7 jours, à partir de l'apparition du 1er plant de Striga hermonthica).

Analyses statistiques: Des analyses de variances à mesures répétés ont été réalisées sur le nombre de graines germées et de plants de striga observés. En cas de différence significative, elles sont complétées par des tests de comparaison de moyennes avec la méthode de Duncan au seuil de $5 \%$. Ces analyses ont été faites à l'aide du logiciel XLSTAT version 2016.02.27444.

montre bien le développement et la croissance d'une plantule de Striga hermonthica fixant le haustorium primaire sur une racine de sésame.
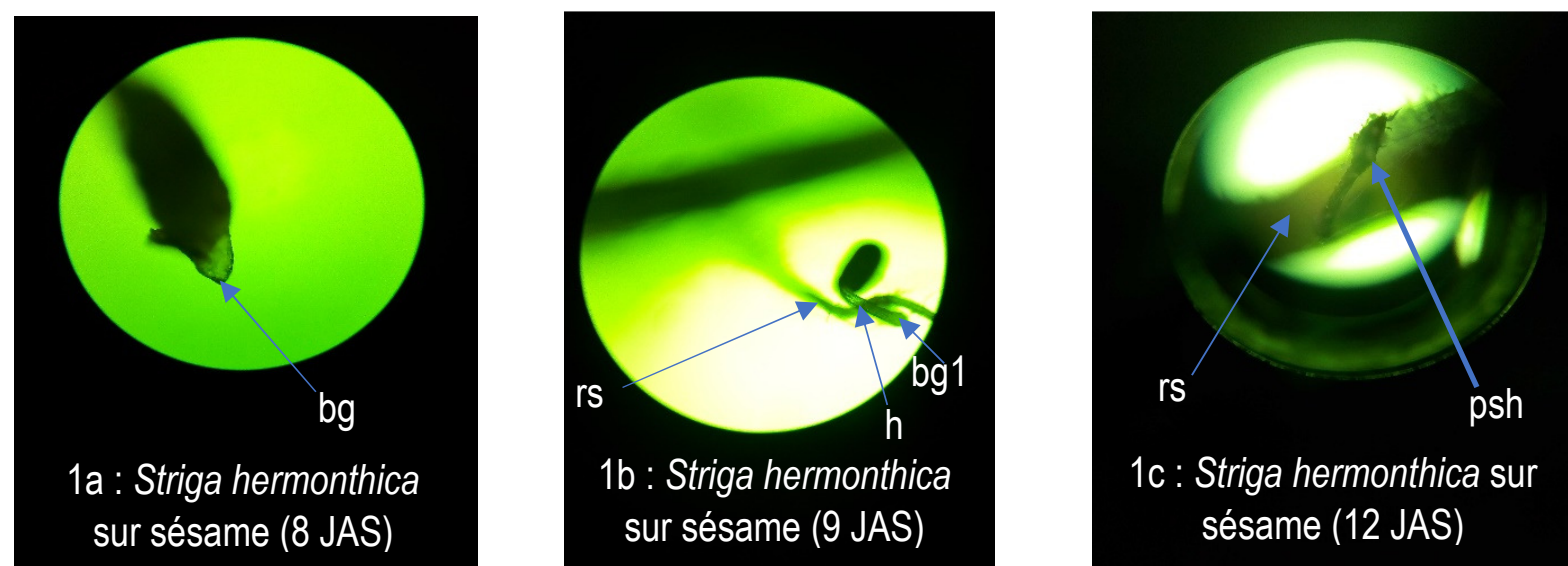

Figure 1: 1a : début de germination d'une graine de Striga hermonthica à 8 jours après semis ; bg : bourgeon germinatif ; $1 \mathrm{~b}$ : formation du haustorium d'une plantule de Striga hermonthica à 9 jours après semis ; rs : racine de sésame ; $\mathrm{h}$ : haustorium ; bg1 : prolongement du bourgeon germinatif ; $1 \mathrm{c}$ : croissance et développement de la plantule de Striga hermonthica à 12 jours après semis ; psh : plantule de Striga hermonthica en croissance et développement et rs : racine de sésame.

L'analyse de variance montre une différence significative pour le nombre de graines de Striga hermonthica germées, avec les probabilités 0,$03 ; 0,01$ et 0,01 (au seuil de $5 \%$ ) respectivement à tous is stades d'observation (4, 8 et 11 JAS). La comparaison des moyennes (tableau 1) montre que l'association de sésame + mil dans la même boite de pétri diminue le taux de germination de graines de Striga hermonthica par rapport au mil pur. A partir de l'observation médiane, l'association mil + Sésame + Striga hermonthica diminue de moitié le taux de germination des graines de Striga hermonthica par rapport au témoin (4 graines germées contre 8 graines germées). A la dernière observation, cette diminution est de 5 graines germées pour l'association contre 12 graines germées pour le témoin. Bien que le sésame fasse germer les graines de Striga hermonthica en boite de pétri, le taux de germination est nettement inférieur aux taux de germinations des autres traitements. A partir de l'observation médiane, les taux de germination des graines de Striga hermonthica sont de même ordre de grandeur pour le traitement sésame pur que pour l'association, mais tous les deux inférieurs à ceux du témoin (mil pur). 
Tableau 1 : Comparaison des moyennes de nombres de graines germées de Striga hermonthica.

\begin{tabular}{|c|c|c|c|c|c|c|c|c|}
\hline \multicolumn{3}{|c|}{ 1ere Observation (4 JAS) } & \multicolumn{3}{|c|}{ Observation médiane (8 JAS) } & \multicolumn{3}{|c|}{ Dernière observation (11 JAS) } \\
\hline Traitements & $\begin{array}{l}\text { Moyennes } \\
\text { (nombres de } \\
\text { graines } \\
\text { germées) et } \\
\text { écart-type }\end{array}$ & Groupes & Traitements & $\begin{array}{l}\text { Moyennes } \\
\text { (nombres de } \\
\text { graines } \\
\text { germées) et } \\
\text { écart-type }\end{array}$ & Groupes & Traitements & $\begin{array}{l}\text { Moyennes } \\
\text { (nombres de } \\
\text { graines } \\
\text { germées) et } \\
\text { écart-type }\end{array}$ & Groupes \\
\hline Mil + Striga & $4 \pm 1,87$ & A & Mil + Striga & $8 \pm 1,98$ & A & Mil + Striga & $12 \pm 1,65$ & A \\
\hline Mil + Sésame + Striga & $3 \pm 2,06$ & A & Mil + Sésame + Striga & $4 \pm 0,82$ & $B$ & Mil + Sésame + Striga & $5 \pm 1,47$ & $\mathrm{~B}$ \\
\hline Sésame + Striga & $0 \pm 0,00$ & $B$ & Sésame + Striga & $3 \pm 0,43$ & $B$ & Sésame + Striaa & $3 \pm 1,73$ & $B$ \\
\hline
\end{tabular}

Tableau 2 : Comparaison et classification des moyennes de plants de Striga hermonthica émergés par traitement et par observation.

\begin{tabular}{|c|c|c|c|c|c|c|c|c|}
\hline \multicolumn{3}{|c|}{ 1ere Observation : 42 JAS } & \multicolumn{3}{|c|}{ Observation médiane : $63 \mathrm{JAS}$} & \multicolumn{3}{|c|}{ Dernière observation : 84 JAS } \\
\hline Traitements & $\begin{array}{l}\text { Moyennes (nombres de } \\
\text { plants) et écart-type }\end{array}$ & Groupes & Traitements & $\begin{array}{l}\text { Moyennes (nombres de } \\
\text { plants) et écart-type }\end{array}$ & Groupes & Traitements & $\begin{array}{l}\text { Moyennes (nombres de } \\
\text { plants) et écart-type }\end{array}$ & Groupes \\
\hline Mil & $1 \pm 0,0$ & A & 38-1-7+Mil & $20 \pm 1,25$ & A & Vgr156+Mil & $71 \pm 2,05$ & A \\
\hline HB168+Mil & $0 \pm 0,0$ & $B$ & Mil & $14 \pm 3,30$ & $A B$ & Mil & $54 \pm 3,56$ & $A B$ \\
\hline EF146+Mil & $0 \pm 0,0$ & $\mathrm{~B}$ & Vgr156+Mil & $13 \pm 2,16$ & $A B$ & ICN137+Mil & $52 \pm 3,30$ & $A B$ \\
\hline HC108+Mil & $0 \pm 0,0$ & $\mathrm{~B}$ & ICN137+Mil & $11 \pm 2,36$ & $A B C$ & HC108+Mil & $49 \pm 6,34$ & $A B$ \\
\hline ICN137+Mil & $0 \pm 0,0$ & $\mathrm{~B}$ & HC108+Mil & $9 \pm 1,41$ & $A B C$ & 38-1-7+Mil & $45 \pm 6,94$ & $\mathrm{ABC}$ \\
\hline HC110+Mil & $0 \pm 0,0$ & $B$ & HB168+Mil & $5 \pm 0,94$ & $\mathrm{BC}$ & EF146+Mil & $42 \pm 3,74$ & $\mathrm{BCD}$ \\
\hline EF147+Mil & $0 \pm 0,0$ & $B$ & Birkan+Mil & $4 \pm 1,70$ & $\mathrm{BC}$ & HB168+Mil & $19 \pm 3,40$ & $\mathrm{CDE}$ \\
\hline Vgr156+Mil & $0 \pm 0,0$ & $\mathrm{~B}$ & EF146+Mil & $4 \pm 1,63$ & $\mathrm{BC}$ & Birkan+Mil & $17 \pm 4,99$ & $\mathrm{DE}$ \\
\hline ICN130+Mil & $0 \pm 0,0$ & $B$ & EF147+Mil & $3 \pm 2,62$ & $\mathrm{BC}$ & EF147+Mil & $16 \pm 3,86$ & $\mathrm{DE}$ \\
\hline 38-1-7+Mil & $0 \pm 0,0$ & $\mathrm{~B}$ & HC110+Mil & $3 \pm 0,82$ & $\mathrm{BC}$ & HC110+Mil & $14 \pm 2,62$ & $\mathrm{E}$ \\
\hline Birkan+Mil & $0 \pm 0,0$ & $\mathrm{~B}$ & ICN130+Mil & $1 \pm 0,47$ & $\mathrm{BC}$ & ICN130+Mil & $9 \pm 0,82$ & $E$ \\
\hline EF146 & $0 \pm 0,0$ & $\mathrm{~B}$ & $\mathrm{HC} 108$ & $0 \pm 0,0$ & C & Birkan & $0 \pm 0,0$ & $E$ \\
\hline HC108 & $0 \pm 0,0$ & B & HB168 & $0 \pm 0,0$ & C & HC108 & $0 \pm 0,0$ & $\mathrm{E}$ \\
\hline ICN137 & $0 \pm 0,0$ & $B$ & EF147 & $0 \pm 0,0$ & $C$ & ICN137 & $0 \pm 0,0$ & $E$ \\
\hline HC110 & $0 \pm 0,0$ & $B$ & Vgr156 & $0 \pm 0,0$ & $C$ & HC110 & $0 \pm 0,0$ & $E$ \\
\hline HB168 & $0 \pm 0,0$ & $B$ & ICN137 & $0 \pm 0,0$ & $C$ & EF147 & $0 \pm 0,0$ & $E$ \\
\hline EF147 & $0 \pm 0,0$ & $B$ & Birkan & $0 \pm 0,0$ & C & Vgr156 & $0 \pm 0,0$ & $E$ \\
\hline Vgr156 & $0 \pm 0,0$ & $B$ & HC110 & $0 \pm 0,0$ & $C$ & ICN130 & $0 \pm 0,0$ & $E$ \\
\hline ICN130 & $0 \pm 0,0$ & $B$ & ICN130 & $0 \pm 0,0$ & $C$ & $38-1-7$ & $0 \pm 0,0$ & $E$ \\
\hline $38-1-7$ & $0 \pm 0,0$ & B & $38-1-7$ & $0 \pm 0,0$ & C & EF146 & $0 \pm 0,0$ & $\mathrm{E}$ \\
\hline Birkan & $0 \pm 0,0$ & $B$ & EF146 & $0 \pm 0,0$ & C & HB168 & $0 \pm 0,0$ & $E$ \\
\hline
\end{tabular}


Le test de germination en pots montre pour l'analyse de variance à mesures répétés, des différences significatives, pour le nombre de plants de Striga hermonthica émergés / pot, pour l'observation médiane (63 JAS) et la dernière observation (84 JAS), avec les probabilités respectives 0,006 et 0,0001 . La différence n'est pas statistiquement significative à la 1ere observation (42 JAS), avec une probabilité de 0,513. La comparaison des moyennes de nombre de plants de Striga hermonthica émergés / pot (tableau 2) montre que l'émergence de Striga hermonthica a commencé à 42 jours après semis, uniquement chez le mil pur (traitement témoin) avec en moyenne 1 plant / pot. La comparaison des moyennes à l'observation médiane montre que le traitement 38-1-7 + Mil (20 plants) provoque plus l'émergence de plants de Striga hermonthica que le traitement témoin (14 plants). La variété Vgr 156 en association avec le mil favorise autant d'émergence (13 plants) de Striga hermonthica que le témoin. En revanche on obtient une diminution de l'émergence des plants de Striga hermonthica avec l'association du mil avec les variétés de sésame

\section{DISCUSSION}

L'étude portée sur le test de germination de Striga hermonthica, par utilisation des exsudats racinaires des légumineuses a donné naissance à l'appellation de plante « faux hôte », toutes les plantes qui provoquent la germination sans permettre le développement du parasite (Andrews, 1947). Striga hermonthica germe aussi bien sur le mil que sur le sésame (faux hôte). Le taux de germination de Striga hermonthica varie de $60 \%$ sur le mil à $15 \%$ sur le sésame. Cependant quand les deux espèces sont associées, ce taux de germination n'est que de $25 \%$. Un taux de germination de Striga hermonthica plus faible $(5 \%)$ est obtenu par l'utilisation d'une autre plante faux hôte, Polygala rarifolia $D C$ en association avec le mil, par Ouédraogo et al., (2018). Des essais sur l'induction de la germination suicide de Striga hermonthica montrent que même les exsudats des plantes faux hôtes (légumineuses), induisent la germination de ces graines (Siame et al., 1993; Abbasher et al., 2019 ; Dafaallah, 2019 ;). Les faux hôtes de Striga hermonthica, les plus utilisés en Afrique de l'Ouest sont : le soja, l'arachide, le cotton, le niébé et le pois de terre (Ramaiah, 1981 ; Parkinson et al., 1986

\section{CONCLUSION ET APPLICATION DES RÉSULTATS}

Cette étude a montré que Striga hermonthica est une plante parasite qui germe aussi bien sur le mil que le sésame considéré comme plante faux hôte (plante suivantes : ICN137 (11 plants), HC108 (9 plants), HB168 (5 plants), Birkan (4 plants), EF 146 (4 plants), EF147 (3 plants) et $\mathrm{HC110}$ (3 plants). ICN130 est la variété qui en association avec le mil a un effet meilleur inhibiteur sur l'émergence (seulement 1 plant à 63 jours après semis contre 14 plants pour le témoin) de Striga hermonthica. A la dernière observation, la variété $\operatorname{Vgr} 156$ en association avec le mil favorise plus l'émergence de Striga hermonthica que même le mil pur. Les variétés ICN137 et HC108 font émerger autant de plants de Striga hermonthica que le témoin (mil pur). On observe une légère diminution de l'émergence des plants de Striga hermonthica avec les associations du mil et des variétés 38-1-7, EF146, HB168, Birkan et EF147. Cette diminution est plus importante avec les variétés $\mathrm{HC} 110$ et ICN130 en association avec le mil (avec seulement respectivement 14 et 9 plants émergés). Cela atteste un effet variétal de sesame très important dans l'expression du phénomène. Cependant, aucune émergence de plant de Striga hermonthica n'est obtenue au niveau des pots de culture pure de sésame contrairement à ce qui est observé dans le test de germination en boite de pétri.

; Yohan et al., 1993). Dans le cas du sésame, on observe uniquement la formation de l'haustorium primaire. Ouédraogo et al., (2018) observe le même résultat en utilisant Polygala rarifolia $D C$. Cette étude vient de montrer que la culture du mil en association avec le sésame, diminue de moitié le nombre de graines germées de Striga hermonthica par rapport à la culture pure du mil. Un effet similaire de réduction de $57 \%$ du nombre de graines de Striga hermonthica germé est observé avec l'utilisation de la fumure organique chez le mil (Daoud et al., 2018 ; Kadri et al., 2019). Toutefois, un effet variétal important est observé chez le sésame. Si les variétés $\mathrm{HC} 110$ et ICN130 utilisées, en association avec le mil, diminuent considérablement le nombre de plants de Striga hermonthica germé / pot, d'autres variétés semblent le favoriser (Vgr 156 et 38-1-7). Enfin, dans le cadre de la lutte contre ce fléau, certains travaux rapportent que l'utilisation de strigolactone en absence de plante hôte donne de résultats similaires en déclenchant la germination suicide du Striga (Gressel, 2009 ; Van Mourik et al., 2011; Kgosi et al., 2012b; Kountche et al., 2019).

piège). L'association du mil + sésame réduit de moitié le nombre de graines germées de Striga hermonthica, par rapport à la culture pure du mil mais, tout dépend de la 
variété de sésame utilisé, car il existe un effet variétal très significatif. Deux variétés de sésame $\mathrm{HC} 110$ et ICN130, ont donné une meilleure efficacité en association avec le mil dans la lutte contre Striga

\section{REMERCIEMENTS}

Ces travaux ont été réalisés dans le cadre de l'élaboration d'une thèse. C'est aussi l'une des activités du projet « Amélioration de la productivité et valorisation du sésame (Sesamum indicum L.) au Niger » financé par le Programme Productivité Agricole en Afrique de

\section{BIBLIOGRAPHIE}

Abbasher, A. A., Zaroug, M. S., Zahran, E. B., \& Sauerborn, J. (2019). Germination, attachment and development of Striga hermonthica (Del.) Benth induced by host and non-host crops. University of Khartoum Journal of Agricultural Sciences, 20(3).

Andrews, F.W., 1947. The parasitism of Striga hermonthica benth. on leguminous plants. Ann. Appl. Biol. 34, 267-275. https://doi.org/10.1111/j.17447348.1947.tb06362.x

Bamba, B., Gueye, M., Ngom, D., Ka, S.L., Diop, B., 2019. Caractérisation des pratiques locales du mil Sanio [Pennisetum glaucum (L.) R. Br] en zone soudanienne humide au Sénégal 10.

Boureima, S., Oukarroum, A., Diouf, M., Cisse, N., Van Damme, P., 2012. Screening for drought tolerance in mutant germplasm of sesame (Sesamum indicum) probing by chlorophyll a fluorescence. Environ. Exp. Bot. 81, 37-43. https://doi.org/10.1016/j.envexpbot.2012.02.01 5

Boussim, I., Yonli, D., Guinko, S., Salle, G., 2012. Etat d'infestation, connaissance endogène et approche systématique des espèces du genre Striga au Burkina Faso. Int. J. Biol. Chem. Sci. 5. https://doi.org/10.4314/ijbcs.v5i4.4

Daoud, M.B., Traore, H., Pale, S., 2018. Effets du système de culture et de la fertilisation organique sur l'infestation de Striga hermonthica et des rendements du maïs et du niébé dans la région du Chari-Baguirmi au Tchad. Int. J. Biol. Chem. Sci. 12, 1260. https://doi.org/10.4314/ijbcs.v12i3.15

Faculty of Agricultural Sciences, University of Gezira, Dafaallah, A.B., 2019. Host Specificity of Sudan Witchweed (Striga hermonthica [Del.] Benth.) Seed Germination and Haustorium Initiation in hermonthica. Ces résultats peuvent être vulgarisés en milieu paysan pour diminuer efficacement le stock de graines de Striga hermonthica dans les champs fortement infestés.

l'Ouest (PPAAO). Nous réitérions nos sincères remerciements à la Faculté d'Agronomie, à l'Institut de la Radio Isotope de l'Université Abdou Moumouni de Niamey et à tous ceux qui ont participé à ces travaux.

Response to Sorghum Root Exudates and Extracts. Int. J. Innov. Approaches Agric. Res. 3 , 287-298. https://doi.org/10.29329/ijiaar .2019 .194 .14

Gressel, J., 2009. Crops with target-site herbicide resistance for Orobanche and Striga control. Pest Manag. Sci. 65, 560-565. https://doi.org/10.1002/ps.1738

Kadri, B.K., Halilou, H., Karimou, I., 2019. Culture du mil [Pennisetum glaucum (L) R. Br] et ses contraintes à la production : une revue. Int. J. Biol. Chem. Sci. 13, 503. https://doi.org/10.4314/ijbcs.v13i1.40

Kgosi, R.L., Zwanenburg, B., Mwakaboko, A.S., Murdoch, A.J., 2012a. Strigolactone analogues induce suicidal seed germination of Striga spp. in soil: Stimulants for suicidal germination of Striga spp. seeds. Weed Res. 52, 197-203. https://doi.org/10.1111/j.13653180.2012.00912.x

Kgosi, R.L., Zwanenburg, B., Mwakaboko, A.S., Murdoch, A.J., 2012b. Strigolactone analogues induce suicidal seed germination of Striga spp. in soil: Stimulants for suicidal germination of Striga spp. seeds. Weed Res. 52, 197-203. https://doi.org/10.1111/j.13653180.2012.00912.x

Kokubun, M., 2018. Crop production under stressful conditions : application of cutting-edge science and technology in developing countries. Springer Berlin Heidelberg, New York, NY.

Kountche, B.A., Jamil, M., Yonli, D., Nikiema, M.P., Blanco-Ania, D., Asami, T., Zwanenburg, B., Al-Babili, S., 2019. Suicidal germination as a control strategy for Striga hermonthica (Benth.) in smallholder farms of sub-Saharan Africa. PLANTS PEOPLE PLANET 1, 107-118. https://doi.org/10.1002/ppp3.32 
Lawane, G., Sougnabé, S.P., Lendzemo, V., Gnokreo, F., Djimasbeye, N., Ndoutamia, G., 2009. Efficacité de l'association des céréales et du niébé pour la production de grains et la lutte contre Striga hermonthica (Del.) 9.

Olivier, A., 1996. La relation entre le Striga hermonthica et ses hôtes : une synthèse. Can. J. Bot. 74, 1119-1137. https://doi.org/10.1139/b96-137

Olivier, A., 1995a. Le striga, mauvaise herbe parasite des céréales africaines: biologie et méthodes de lutte. Agronomie 15, 517-525. https://doi.org/10.1051/agro:19950901

Olivier, A., 1995b. Le striga, mauvaise herbe parasite des céréales africaines: biologie et méthodes de lutte. Agronomie 15, 517-525. https://doi.org/10.1051/agro:19950901

Olivier, A., n.d. La relation entre Striga hermonthica et ses hôtes : une synthèse 20.

Olupot, J.R., Osiru, D.S.O., Oryokot, J., Gebrekidan, B., 2003. The effectiveness of Celosia argentia (Striga "chaser") to control Striga on Sorghum in Uganda. Crop Prot. 22, 463-468. https://doi.org/10.1016/S0261-2194(02)001813

Ouédraogo, O., Kaboré, T.D., Noba, D.R., Traoré, S., 2018. Polygala rarifolia DC., plante faux hôte du Striga hermonthica (Del.) Benth. J. Appl. Biosci. 123 , 12346. https://doi.org/10.4314/jab.v123i1.3

Rath, A.C., Kang, I.-K., Park, C.-H., Yoo, W.-J., Byun, J.K., 2006. Foliar application of aminoethoxyvinylglycine (AVG) delays fruit ripening and reduces pre-harvest fruit drop and ethylene production of bagged "Kogetsu" apples. Plant Growth Regul. https://doi.org/10.1007/s10725-006-0009-3

Siame, B. A., Weerasuriya, Y., Wood, K., Ejeta, G., \& Butler, L. G. (1993). Isolation of strigol, a germination stimulant for Striga asiatica, from host plants. Journal of Agricultural and Food Chemistry, 41(9), 1486-1491.

Showemimo, F. A., Kimbeng, C. A., \& Alabi, S. O. (2002). Genotypic response of sorghum cultivars to nitrogen fertilization in the control of Striga hermonthica. Crop Protection, 21(9), 867-870.

Spallek, T., Mutuku, M., Shirasu, K., 2013. The genus Striga: a witch profile: Profile of Striga, the witchweed. Mol. Plant Pathol. 14, 861-869. https://doi.org/10.1111/mpp.12058
Stoop, W. A., Lanting, H., \& Ramaiah, K. V. (1981). Occurrence of Striga and its possible control in cropping systems in Northern Ghana. KV Ramaiah and MJ Vasudeva Rao, 103.

Van Mourik, T.A., Stomph, T.J., Murdoch, A.J., 2011. Purple witchweed (Striga hermonthica) germination and seedbank depletion under different crops, fallow, and bare soil: Striga germination and seedbank depletion. Weed Biol. Manag. 11, 100-110. https://doi.org/10.1111/j.14456664.2011.00409.x

Waters, M.T., Gutjahr, C., Bennett, T., Nelson, D.C., 2017. Strigolactone Signaling and Evolution. Annu. Rev. Plant Biol. 68, 291-322. https://doi.org/10.1146/annurev-arplant042916-040925

Zia, S., Khan, M.A., 2004. Effect of light, salinity, and temperature on seed germination of Limonium stocksii. Can. J. Bot. 82, 151-157. https://doi.org/10.1139/b03-118 